\title{
Estimating the national and regional prevalence of drinking or eating more than usual during childhood diarrhea in Malawi using the bivariate sample selection copula regression
}

\author{
Alfred Ngwira ${ }^{\text {Corresp., } 1}$, Francisco Chamera ${ }^{1}$, Matrina Mpeketula Soko \\ ${ }^{1}$ Basic Sciences Department, Lilongwe University of Agriculture and Natural Resources, Lilongwe, Malawi \\ Corresponding Author: Alfred Ngwira \\ Email address: alfngwira@yahoo.com
}

Background. Estimation of prevalence of feeding practices during diarrhea using conventional imputation methods may be biased as these methods apply to observed factors and in this study, feeding practice status was unobserved for those without diarrhea. The study aimed at re-estimating the prevalence of feeding practices using the bivariate sample selection model. Methods. The study used 2015-16 Malawi demographic health survey (MDHS) data which had 16246 children records who had diarrhea or not. A

bivariate Joe copula regression model with 90 degrees rotation was fitted to either drinking or eating more, with diarrhea as a sample selection outcome in the bivariate models. The prevalence of drinking more than usual and prevalence of eating more than usual were then estimated based on the fitted bivariate model. These prevalences were then compared to the prevalences estimated using the conventional imputation method. Results. There was a substantial increase in the re-estimated national prevalence of drinking more fluids $[40.0 \%, 95 \% \mathrm{Cl}=31.7-50.5]$ or prevalence of eating more food [20.46\%, $95 \% \mathrm{Cl}=9.87-38.55]$ using the bivariate model as compared to the prevalences estimated by the conventional imputation method, that is, [28.9\%, $95 \% \mathrm{Cl}=$ 27.0 - 30.7] and [13.1 \% , $95 \% \mathrm{Cl}=12.0$ - 15.0] respectively. The maps of the regional prevalences showed similar results where the prevalences estimated by the bivariate model were relatively higher than those estimated by the standard imputation method. The presence of diarrhea was somehow weakly negatively correlated with either drinking more fluids or eating more food. Conclusion. The estimation of prevalence of drinking more fluids or eating more food during diarrhea should use bivariate modelling to model sample selection variable so as to minimize bias. The observed negative correlation between diarrhea presence and feeding practices implies that mothers should be encouraged to let their children drink more fluids or eat more food during diarrhea episode to avoid dehydration and malnutrition. 


\section{Estimating the national and regional prevalence of drinking}

2 or eating more than usual during childhood diarrhea in

3 Malawi using the bivariate sample selection copula

4 regression.

5 Alfred Ngwira ${ }^{1}$, Francisco Chamera ${ }^{1}$, Matrina Mpeketula Soko ${ }^{1}$

6 'Basic Sciences Department, Lilongwe University of Agriculture and Natural Resources,

7 Lilongwe, Malawi

8 Corresponding Author:

9 Alfred Ngwira ${ }^{1}$

10 Bunda College, Lilongwe, Malawi.

11 Email: alfngwira@yahoo.com 
26 Abstract

27 Background. Estimation of prevalence of feeding practices during diarrhea using conventional imputation methods may be biased as these methods apply to observed factors and in this study, feeding practice status was unobserved for those without diarrhea. The study aimed at reestimating the prevalence of feeding practices using the bivariate sample selection model. Methods. The study used 2015-16 Malawi demographic health survey (MDHS) data which had 16246 children records who had diarrhea or not. A bivariate Joe copula regression model with 90 degrees rotation was fitted to either drinking or eating more, with diarrhea as a sample selection outcome in the bivariate models. The prevalence of drinking more than usual and prevalence of eating more than usual were then estimated based on the fitted bivariate model. These prevalences were then compared to the prevalences estimated using the conventional imputation method.

Results. There was a substantial increase in the re-estimated national prevalence of drinking more fluids $[40.0 \%, 95 \% \mathrm{CI}=31.7$ - 50.5] or prevalence of eating more food $[20.46 \%, 95 \% \mathrm{CI}$ $=9.87-38.55]$ using the bivariate model as compared to the prevalences estimated by the conventional imputation method, that is, $[28.9 \%, 95 \% \mathrm{CI}=27.0-30.7]$ and $[13.1 \%, 95 \% \mathrm{CI}$ $=12.0-15.0]$ respectively. The maps of the regional prevalences showed similar results where the prevalences estimated by the bivariate model were relatively higher than those estimated by the standard imputation method. The presence of diarrhea was somehow weakly negatively correlated with either drinking more fluids or eating more food.

Conclusion. The estimation of prevalence of drinking more fluids or eating more food during diarrhea should use bivariate modelling to model sample selection variable so as to minimize bias. The observed negative correlation between diarrhea presence and feeding practices implies that mothers should be encouraged to let their children drink more fluids or eat more food during diarrhea episode to avoid dehydration and malnutrition.

Key words: under-five, feeding practices, child health, malnutrition, pediatrics 


\section{Introduction}

57 Diarrhea in children is defined as the occurrence of three or more loose or liquid stools per day 58 (WHO, 2013). Diarrhea is considered the second killer of under-five children after pneumonia causing an estimated 1.5 million under-five deaths every year (WHO, 2009). The prevalence of diarrhea in Malawi as of 2016 was 22\% (NSO, 2017). Diarrhea has a detrimental effect on child's nutrition status due to decreased food intake caused by anorexia (Huffman et al, 1991). To reduce deaths, dehydration and minimize the effects of diarrhea on nutritional status, mothers are encouraged to improve on feeding practices especially to give children more fluids and food than usual. The prevalence of feeding practices during childhood diarrhea in Malawi from the latest Malawi demographic health survey (NSO, 2017) report is $31 \%$ for drinking more fluids and $13 \%$ for eating more food.

The estimation of prevalence of feeding practices during childhood diarrhea using demographic health survey (DHS) data is done by focusing on children who had diarrhea in the last two weeks. In this case, the prevalence of either drinking or eating more than usual is the number of children with diarrhea who drink or eat more than usual divided by the total number of children with diarrhea (Croft et al, 2018). The use of complete case analysis (i.e children who had diarrhea only) in the estimation of the feeding practices prevalence is biased since the selected sample is not random, that is, selection is more or less like voluntarily, since only children with diarrhea are considered. In addition, some participants who might have had responded that they did not have diarrhea, who are left out of the sample, might have said so due to fear of being ashamed of the disease particularly wealthier people who think they are always hygienic, while in actual sense they might have the disease and their feeding practice status would be better. This would result in underestimation bias of the prevalence. The use of children with diarrhea only can be considered as a missing data problem, where the feeding practice status is not observed for children without diarrhea. Standard or conventional approaches to dealing with missing data are single imputation, multiple imputation, inverse probability weighting or propensity score reweighting (McGovern et al, 2015). However if such methods can be applied in this study, they can be biased since they only account for observed factors and in this study, feeding practice status is not observed for those without diarrhea (McGovern et al, 2015). An alternative method which yield better estimates than the standard methods mentioned is the use of Heckman-style sample selection models (Kone et al, 2019). In this case, two equations are 
87 used, where one equation models the sample selection variable, for example, diarrhea status (yes/no) and another equation models the outcome variable of interest, for example, feeding practice status, for example, drinking more fluids or eating more food than usual.

Usually, the presence of diarrhea in a child is associated with poor household characteristics, for example, poor parental education according to Asfaha et al (2018) [AOR = 2.88, 95\% CI = $1.70-4.88]$ and Manun'Ebo and Nkulu-wa-Ngoie (2020) [AOR =1.14, 95 \% CI $=0.93-1.40]$, and large household size according to Omona et al $(2020)$ [AOR $=7.185,95 \% \mathrm{CI}$ $=1.353-38.147]$. Such poor household characteristics in turn are associated with poor feeding practices during diarrhea episode, for example, according to Fikadu and Girma (2018), mothers who have one under-five child are 2 times $[\mathrm{AOR}=2.11,95 \% \mathrm{CI}=1.38-3.23]$ more likely to have proper feeding practices during diarrhea episode as compared to those who have two and more under-five children. It is hypothesized therefore that there is a negative association between the presence of diarrhea and feeding practices (drinking or eating more) during childhood diarrhea.

The purpose of this study was to re-estimate the prevalence of drinking or eating more than usual during childhood diarrhea episode using sample selection copula bivariate model. The advantage of copula regression over the standard bivariate normal regression is that it offers flexibility in modelling dependence or association between two outcome variables (Parsa and Klugman, 2011). In this case, the copula regression can even model asymmetric dependence which is not the case with the standard bivariate normal distribution (McGovern et al, 2015). For example, in this study, since the hypothesized association between diarrhea presence and feeding practice status is negative, an exploration of asymmetric copula functions like the Joe copula with 90 degrees rotation which models the negative dependence was made. Specifically, the study sought to find out if diarrhea and drinking or eating more than usual were dependent and also the study aimed at re-estimating the prevalence of eating or drinking more than usual using the sample selection model. The significance of the study is that it would reveal if the estimates of prevalence of eating or drinking more during childhood diarrhea reported in the DHS report (NSO, 2017) were biased or not due to the use of sample with diarrhea only. This would ensure correct policy making using the correct prevalences. 

statistical analysis, are presented. This is followed by presentation of results, discussion and then conclusion.

\section{Materials \& Methods}

\section{Data}

121 The study used secondary data, the 2015-16 Malawi DHS child record data. Permission to use

122 the data was granted after asking for permission to use the data through the DHS web site 123 (www.dhsprogram.com/data set_admin). The 2015-16 MDHS study was ethically approved by 124 Malawi Health Research Committee, Institutional Review Board of ICF Macro, Center for 125 Disease and Control (CDC) in Atlanta, GA, USA. No any other permission was needed to 126 publish the results of the study. The MDHS according to NSO (2017) was a two stage cluster 127 sampling with stratification where clusters were stratified by residence (urban/rural) and then in 128 each cluster, households were randomly selected. In the first stage, 850 clusters, comprising of 129173 clusters of urban areas and 677 clusters of rural areas were selected by probability 130 proportional to size (PPS) cluster sampling method. In the second stage, 30 households from each urban cluster and 33 households from each rural cluster were selected by systematic sampling. The data from households was then collected using the four questionnaires, that is, the woman, man, household and then biomarker questionnaire. This study used the child record data mainly collected by the woman questionnaire. The response variables in the final data set were the presence of child diarrhea (yes/no) in the last two weeks and the feeding practice variables, namely, eating food more than usual (yes/no) and drinking fluids more than usual (yes/no). The independent variables were child age (in months), household size, maternal education (no education, primary, secondary, higher), water source (safe/unsafe), toilet (yes/no), house floor (cement/sand), district code of the child and interviewer identity code. The total number of records in the final data set was 16246 .

\section{Statistical analysis}

142 A frequency distribution table of feeding practice in terms food and fluids intake during

143 childhood diarrhea was made. A multiple variable bivariate sample selection model as defined 144 by Marra and Radice (2017a) was then fitted, that is, if $y_{i 1}$ is the sample selection outcome, that 145 is, diarrhea presence (yes/no) and $y_{i 2}$ is the second outcome observed only if child is selected, 
146 that is, has diarrhea, in this study for example, drinking more fluids or eating more food than

147 usual. Then the flexible additive predictor of the bivariate model of diarrhea presence (yes/no)

148 and drinking more or diarrhea presence (yes/no) and eating more food is defined as

$149 \eta_{v i}=\beta_{v 0}+\sum s_{v k, i}\left(z_{v k, i}\right), i=1,2,3, \ldots, n$ where $v=1,2, c$. In addition, in the predictor, $\beta_{0}$ is the

150 intercept and $s_{v k, i}\left(z_{v k, i}\right)$ represents the generic effect of the independent variable and is specified

151 according to the type of covariate considered. When $v=c$, the additive predictor $\eta_{c i} \quad$ is for the

152 copula parameter denoted by $\theta_{i}$, the dependence parameter between the two outcome variables

153 involved. The bivariate distribution used in this study was the Joe copula with 90 degrees

154 rotation which was opted for so as to model the negative dependence between diarrhea and the

155 feeding practice variables as per the hypothesis, and after the standard bivariate normal copula

156 revealed a negative dependence between the two outcome variables. The range of the copula

157 parameter with this bivariate distribution was $(-\infty,-1)$ where $\theta=-1$ means independence and

158 dependence otherwise. The main objective in this study was to estimate the prevalence of the

159 observed outcome, $y_{i 2}$, given the selected sample, for example, the prevalence of drinking more

160 fluids or eating more food during diarrhoea episode defined as $P\left(Y_{2}=1\right)$. This was

161 computed by the formula: $P\left(Y_{2}=1\right)=\frac{\sum_{i=1}^{n} w_{i}\left[1-F_{2}\left(\hat{\eta}_{i 2}\right)\right]}{\sum_{i=1}^{n} w_{i}}$, where $w_{i}$ were the survey weights, the

162 woman individual sample weights. Model estimation was by penalized maximum likelihood

163 estimation (PMLE) considering that the usual maximum likelihood estimation (MLE) could lead

164 to over fitting due to the presence of smooth functions (Filippou et al, 2017). Model fitting,

165 prevalence estimation and mapping of regional prevalence was implemented by the GJRM

166 package in R (Marra and Radice, 2017b).

167

\section{Results}

169 Table 1 presents the percentage of children under-five in terms of feeding practices during

170 diarrhea as reported from MDHS report (NSO, 2017). Thirty one percent of children with

171 diarrhea were given more than usual fluids and thirty four percent were given less fluids which

172 is a concern. Five percent of the sick children were not given any fluids. Sixty one percent of the

173 sick children were given recommended liquid as compared to fourty five percent children that

174 were given recommended food. These percentages were used as a bench mark as the new 
175 percentages using the sample selection model were estimated. The focus however was on the 176 percentage of children feeding or drinking more than usual during diarrhea.

177 After fitting the bivariate sample selection model using the Joe copula, the estimated

178 national prevalence of drinking more fluids during childhood diarrhea using the conventional

179 imputation method was $28.9 \%$ with $95 \%$ confidence interval as $(27.0,30.7)$. Adjusting for

180 sample selection, using the sample selection bivariate model, the estimated prevalence of

181 drinking more fluids was $40.0 \%$ with $95 \%$ confidence interval $(31.7,50.5)$. Regarding eating

182 more food, the estimated national prevalence of eating more during diarrhea using the

183 conventional imputation method was $13.1 \%$ with $95 \%$ confidence interval $(12.0,15.0)$, and the

184 estimated prevalence using the bivariate sample selection model was $20.46 \%$ with $95 \%$

185 confidence interval $(9.87,38.55)$. The estimated average copula parameter for the bivariate

186 model of diarrhea and drinking more fluids was -1.23 with $95 \%$ confidence interval as (-1.53,-

187 1.09) and that of diarrhea and eating more food was -1.29 with $95 \%$ confidence interval as (-

188 2.57, -1.04), both showing negative dependence. The association of drinking and eating more

189 with diarrhea may be considered as weak since the copula parameter values are close to -1 .

$190 \quad$ Figure 1 presents the map of prevalence of drinking more fluids during childhood

191 diarrhea by region. The prevalence estimates by the bivariate sample selection model (Figure 1B)

192 are relatively higher than the imputation based estimates (Figure 1A). The copula parameter

193 shows relatively strong negative dependence between diarrhea and drinking more fluids in many

194 districts (Figure 1C). Figure 2 shows the distribution of prevalence of eating more food. The

195 prevalence estimates by the bivariate sample selection model (Figure 2B) are also relatively

196 higher than the imputation based estimates (Figure 2A). The copula parameter distribution shows

197 many areas having weak dependence between diarrhea and eating more food (Figure 2C).

198

199 Discussion

200 The study has looked at the re-estimation of the prevalence of feeding practices during childhood

201 diarrhea by focusing on drinking and eating more than usual. The standard estimation procedure

202 is prone to be biased as the final selected sample is not random as individuals are selected

203 depending on whether they have diarrhea or not. The study therefore tried to investigate the

204 degree of biasness attached to the estimates if the standard method is used, by re-estimating the

205 prevalences using the novel approach which takes into account sample selection process. Sample 
206 selection process was taken into account in estimation by using the bivariate sample selection

207 model where one of the two equations, modeled sample selection variable, diarrhea status 208 (yes/no).

The study found that the prevalence estimates of both drinking more liquids and eating 210 more food using the bivariate model were substantially greater than those estimated by the conventional, imputation method. The increased prevalence was likely, since one international study (Bani et al, 2002) found much higher prevalence of mothers increasing the volume of fluids given during childhood diarrhea episode $(75.5 \%)$. The observed differences in the prevalence estimated by the bivariate sample selection model and the usual imputation method might be due to the non-randomness of the sample selected (Marra and Radice, 2017). Nonrandomness of the sample would be as a result of selection process, as samples were being selected if they had diarrhea, otherwise they were not selected. The estimates by bivariate sample selection model would be with minor bias as non-randomness was corrected by modelling sample selection variable (diarrhea status) in addition to modelling the outcomes of interest, feeding status variables. The increase in prevalence estimate from standard estimation to sample selection estimation may be due to the fact that those not selected (without diarrhea) were likely to have good education and small number of children since low education and large household size are positively correlated with diarrhea (Asfaha et al, 2018) and this would mean increased drinking and eating more in case sample selection process is corrected as increased education and small household size increase the intake of fluids and food (Fikadu and Girma, 2018). An indication of weak correlation between drinking and eating more food and diarrhea is consistent with Huffman et al (1991) where having diarrhea did not significantly affect eating habits though there was a decline in eating food as children appetite reduced.

The distribution of estimated prevalence of drinking or eating more by region (Figure 1 $\& 2, \mathrm{~b})$ shows that there is less variation and most regions especially around the cities show increased intake of fluids and food. Generally, there is reduced intake of food compared to fluids. The reduction in food intake may be due to decrease in appetite in the diarrhoea patients as explained by Huffman et al (1991), and Paintal and Aguayo (2016). Similar distribution of fluid and food intake across all the regions may due to the fact that all regions might have similar spatial determinants of feeding practices during diarrhea, for example, average household size is 
237 sizes: 3.7 (north), 3.6 (central) and 3.7 (south) (NSO, 2017), and household size has been found

238 to be associated with feeding practices (Fikadu and Girma, 2018).

239

\section{Conclusion}

241 The study finds that the both estimated prevalence of drinking and eating more food by the

242 standard method were likely to be biased considering that they deviated greatly from the estimate

243 based on the sample selection model found in this study. There is weak negative correlation

244 between the presence of diarrhea and the feeding practices during diarrhea. The implication of

245 the results is that the prevalence of drinking or eating more food during childhood diarrhea

246 should be estimated by taking into account sample selection process (diarrhea presence) so as to

247 correct for the biasness that may arise due to non-randomness of the sample.

\section{Acknowledgements}

249 We thank the demographic health survey (DHS) for providing the data that was used.

250

251

252

253

254

255

256

257

258

259

260

261

262

263

264

\section{References}

1. WHO. 2013. Diarrheal diseases: World Health Organization. Available at: https://www.who.int/news-room/fact-sheets/detail/diarrhoeal-disease

2. UNICEF/WHO. 2009. Diarrhoea: Why children are still dying and what can be done. Available at: https://www.who.int/maternal_child_adolescent/documents/9789241598415/en/

3. National Statistical Office (NSO) [Malawi] and ICF. 2017. Malawi Demographic and Health Survey 2015-16. Available at: https://dhsprogram.com/pubs/pdf/FR319/FR319.pdf

4. Huffman SL, Romana GL, Brown KH, Bentley M, Black RE. 1991. Do Child Feeding Practices Change in the Central Peruvian Highlands. J Diarrhoeal Dis Res 9(4): 295-300.

5. Croft, Trevor N, Marshall AMJ, Allen CK. 2018. Guide to DHS Statistics. Rockville, Maryland, USA: ICF. Available at: https://dhsprogram.com/pubs/pdf/DHSG1/Guide_to_DHS_Statistics_DHS-7_v2.pdf

6. McGovern ME, Bärnighausen T, Marra G, Radice R. 2015. On the Assumption of Bivariate Normality in Selection Models: A Copula Approach Applied to Estimating HIV Prevalence. Epidemiology 26(2): 229-237 
265 7. Koné S, Bonfoh B, Dao D, Koné I, Fink G. 2019. Heckman-type selection models to obtain

266

267

268

269

270

271

272

273

274

275

276

277

278

279

280

281

282

283

284

285

286

287

288

289

290

291

292

293

294

295 unbiased estimates with missing measures outcome: theoretical considerations and an application to missing birth weight data. BMC Medical Research Methodology 19:231.

8. Asfaha KF, Tesfamichael FA, Fisseha GK, Misgina KH, Weldu MG, Welehaweria NB, Gebregiorgis YS. 2018. Determinants of childhood diarrhoea in Medebay Zana District, Northwest Tigray, Ethiopia: a community based unmatched case-control study. BMC Pediatrics 18:120.

9. Manun'Ebo MF, Nkulu-wa-Ngoie C. 2020. Factors associated with child's comorbid diarrhea and pneumonia in rural Democratic Republic of the Congo. African Journal of Medical and Health Sciences 19(5): 55-62.

10. Omona S, Malinga GM, Opoke R, Openy G, Opiro R. 2020. Prevalence of diarrhoea and associated risk factors among children under five years old in Pader District, northern Uganda. BMC Infectious Diseases 20:37

11. Fikadu T, Girma S. 2018. Feeding Practice during Diarrheal Episode among Children Aged between 6 to 23 Months in Mirab Abaya District, Gamo Gofa Zone, Southern Ethiopia. International Journal of Pediatrics 18.

12. Parsa RA, Klugman SA. 2011. Copula Regression. Variance 5(1): 45-54.

13. Marra G, Radice R. 2017a. A joint regression modeling framework for analyzing bivariate binary data in R. De Gruyter Open 5: 268-294

14. Filippou P, Marra G, Radice R. 2017. Penalized likelihood estimation of a trivariate additive probit model. Biostatistics 18(3): 569-585

15. Marra G, Radice R. 2017b. GJRM: Generalised Joint Regression Modelling. R package version 0.1-2. Available on CRAN.

16. Bani IA, Saeed AAW, Mohammed AA, Othman A. 2002. Diarrhoea and child feeding practices in Saudi Arabia. Public Health Nutrition 5(6), 727-731

17. Paintal K, Aguayo VM. 2016. Feeding practices for infants and young children during and after common illness. Evidence from South Asia. Maternal \& Child Nutrition 12 (1): 39-71 
296 Table 1: Percentage of feeding practices during childhood diarrhea

297 Figure 1: Prevalence map of drinking more fluids by district. (A) Imputation model. (B) sample 298 selection model. (C) copula parameter. Darker (high), gray (middle) and white (low).

299 Figure 2: Prevalence map of eating more food. (A) Imputation model. (B) sample selection 300 model. (C) copula parameter. Darker (high), gray (middle) and white (low).

301 
Table $\mathbf{1}$ (on next page)

Percentage of feeding practices during diarrhoea 
1 Table 1: Percentage of feeding practices during diarrhoea

\begin{tabular}{|l|l|l|l|l|l|}
\hline & \multicolumn{5}{|c|}{ Feeding practice } \\
\hline & more & usual/same & less & none & never gave \\
\hline Liquids & 31 & 30 & 34 & 5 & 0 \\
\hline Food & 13 & 32 & 43 & 6 & 5 \\
\hline
\end{tabular}

2 


\section{Figure 1}

Prevalence map of drinking more fluids by district. (A) Imputation model. (B) Sample selection model. (C) Copula parameter. Darker (high), gray (middle) and white (low).
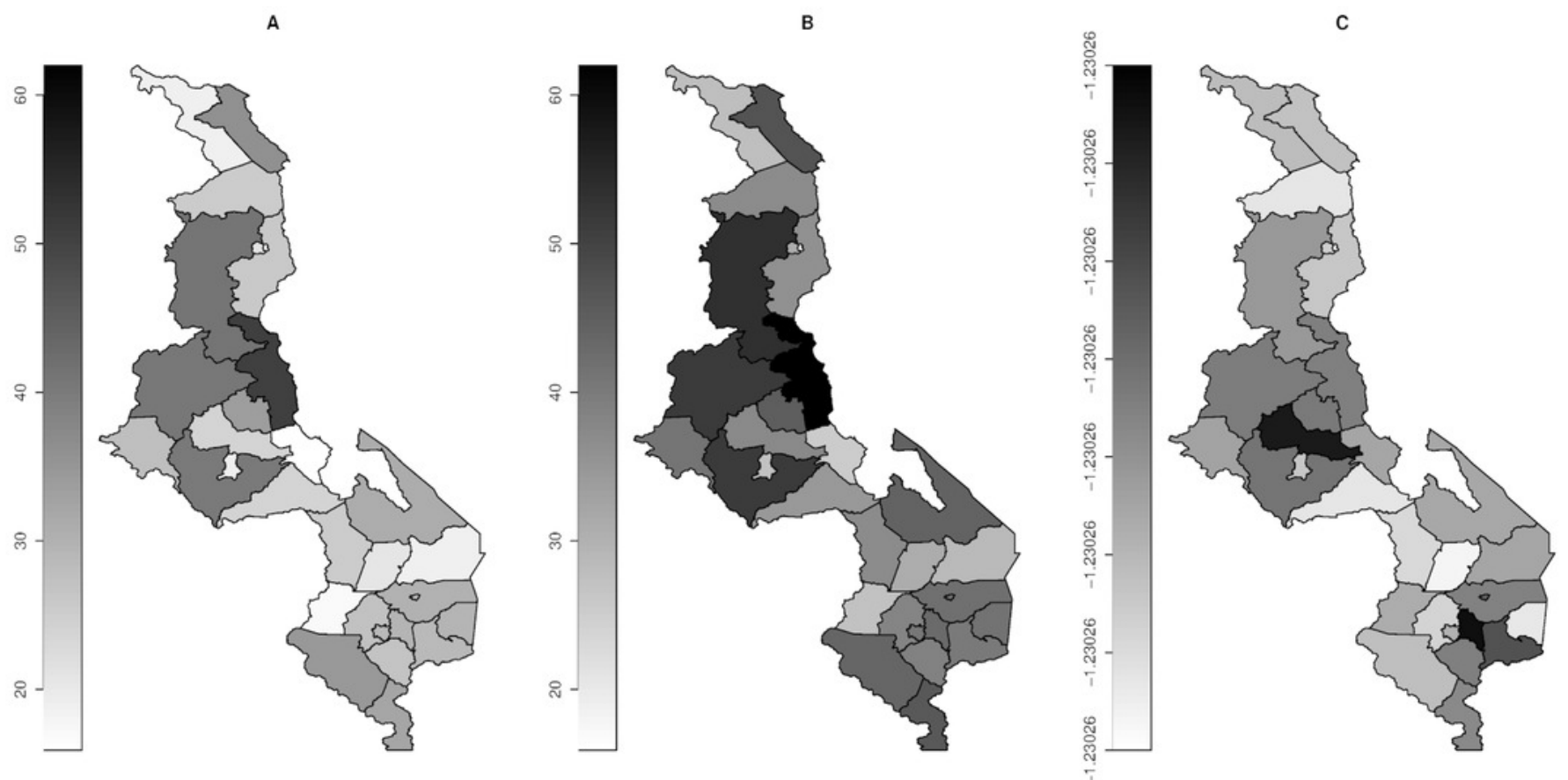
Figure 2

Prevalence map of eating more food. (A) Imputation model. (B) Sample selection model. (C) Copula parameter. Darker (high), gray (middle) and white (low).

A

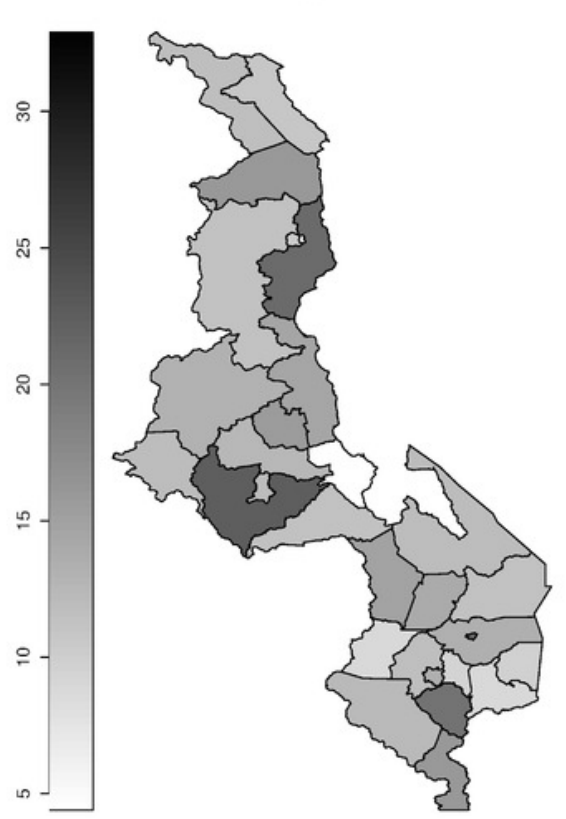

B

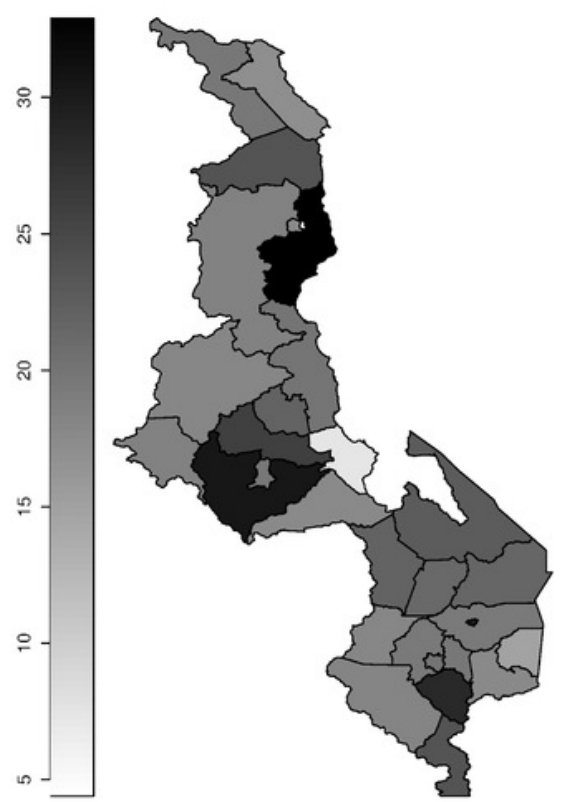

c

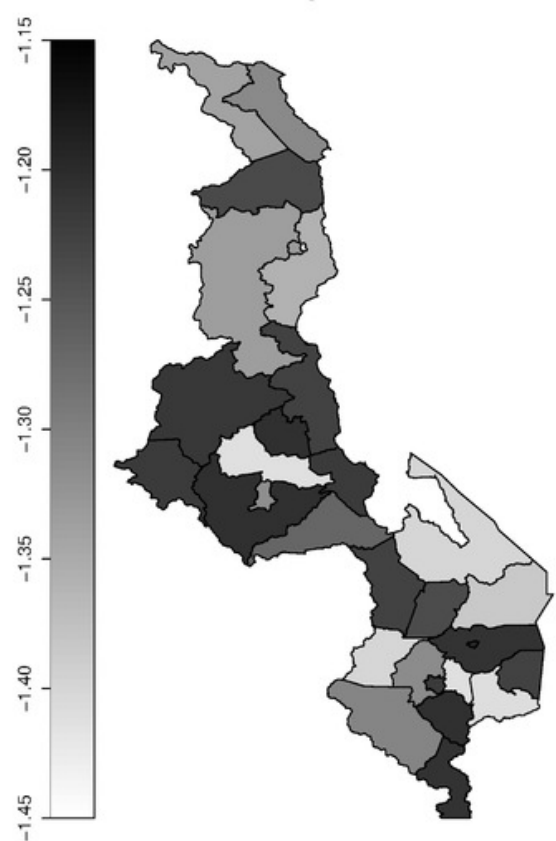

